\title{
Propriétés et utilisation de I'huile de ricin
}

\section{Franck DUMEIGNIL}

Université Lille Nord de France, F-59000,

Lille,

France ;

CNRS UMR8181,

Unité de Catalyse et Chimie du Solide,

UCCS,

F-59655 Villeneuve d'Ascq, France;

Institut Universitaire de France,

10, Boulevard Saint-Michel,

75005 Paris,

France

$<$ franck.dumeignil@univ-lille1.fr>

\begin{abstract}
Castor and its derived oil, the so-called "castor oil", are known from the ancient times, when the oil was used for cosmectics, or as a laxative, or as a fuel in lamps. Nowadays, castor oil has a completely renewed interest, not for its direct applications, but thanks to various possibilities in downstream processing, which involves chemical modifications \& reactions. Indeed, castor oil has this specifiticy of a remarkable uniformity, with about $90 \%$ of the fatty acid chains of the triglyceride composed of ricinoleic acid, a natural fatty acid behaving a hydroxyle group, which is a unique feature in vegetal oils. Then, it is possible to envision reactions either on the ester group, on the double bond or even on the hydroxyle moety to yield a variety of chemicals. In this paper, after a short description of the castor plant, we give statistics on the production of castor grains and an overview on the oil extraction process. Then, we report the market prices before listing the main properties of castor oil. These latter are quite specific and explain the large variety of possible direct applications. At last, the possible chemical transformations are listed, before giving two practical cases, as actual industrialisation success stories.
\end{abstract}

Key words: castor, castor oil, ricinoleic acid, lubricant, pharmacy, food, chemicals, applications

ricin était aussi dédiée à des usages cosmétiques : on rapporte que Cléopâtre en utilisait une goutte pour faire briller le blanc de ses yeux et s'en servait aussi comme démaquillant. Ses vertus laxatives étaient également déjà connues dans l'Égypte ancienne, puisqu'elles sont par ailleurs décrites dans l'Ebers Papyrus, I'un des plus anciens traités médicaux jamais recensés, daté d'environ 1550 avant Jésus-Christ. C'est aussi dans ces temps anciens, vers 2000 ans avant Jésus-Christ, que l'on retrouve trace d'usages équivalents de l'huile de ricin en Inde, comme combustible dans les lampes à huile, en médecine comme laxatif, mais aussi comme composé de choix pour la guérison des arthroses selon la médecine ayurvédique. La Chine s'est par ailleurs aussi intéressée, un peu plus tard semble-t-il, au ricin dont elle a importé la culture depuis I'Inde il y a environ 1400 ans, à l'époque pour des usages médicaux mais aussi culinaires. Plus récemment, les propriétés extrêmement toxiques du ricin ont été exploitées à des fins coercitives pendant les heures noires de I'Italie sous la dictature de Mussolini. Dans la même veine d'usage à des fins peu glorieuses, on peut aussi mentionner, par exemple, le tristement célèbre coup du « parapluie bulgare » en pleine guerre froide à Londres en 1978, lors duquel l'écrivain dissident bulgare Georgi Markov s'est vu administrer en pleine rue une dose létale de ricine dans le mollet à l'aide d'un parapluie à l'embout trafiqué. On ne sera ainsi pas étonné que I'huile de ricin soit classée parmi les huiles dites "non alimentaires ». Les propriétés agronomiques de la plante de ricin qui offre d'importants rendements en huile, sont un facteur supplémentaire qui légitimise l'huile de ricin comme molécule de base très intéressante pour des utilisations dans le domaine de la chimie.

\section{Production}

Le ricin est une plante qui s'est à l'origine développée en Égypte, en Éthiopie et en Inde mais dont la culture s'est ensuite étendue à de nombreux autres pays. La

Pour citer cet article : Dumeignil F. Propriétés et utilisation de I'huile de ricin. OCL 2012 ; 19(1) : 10-15. doi : 10.1684/ocl.2012.0427 


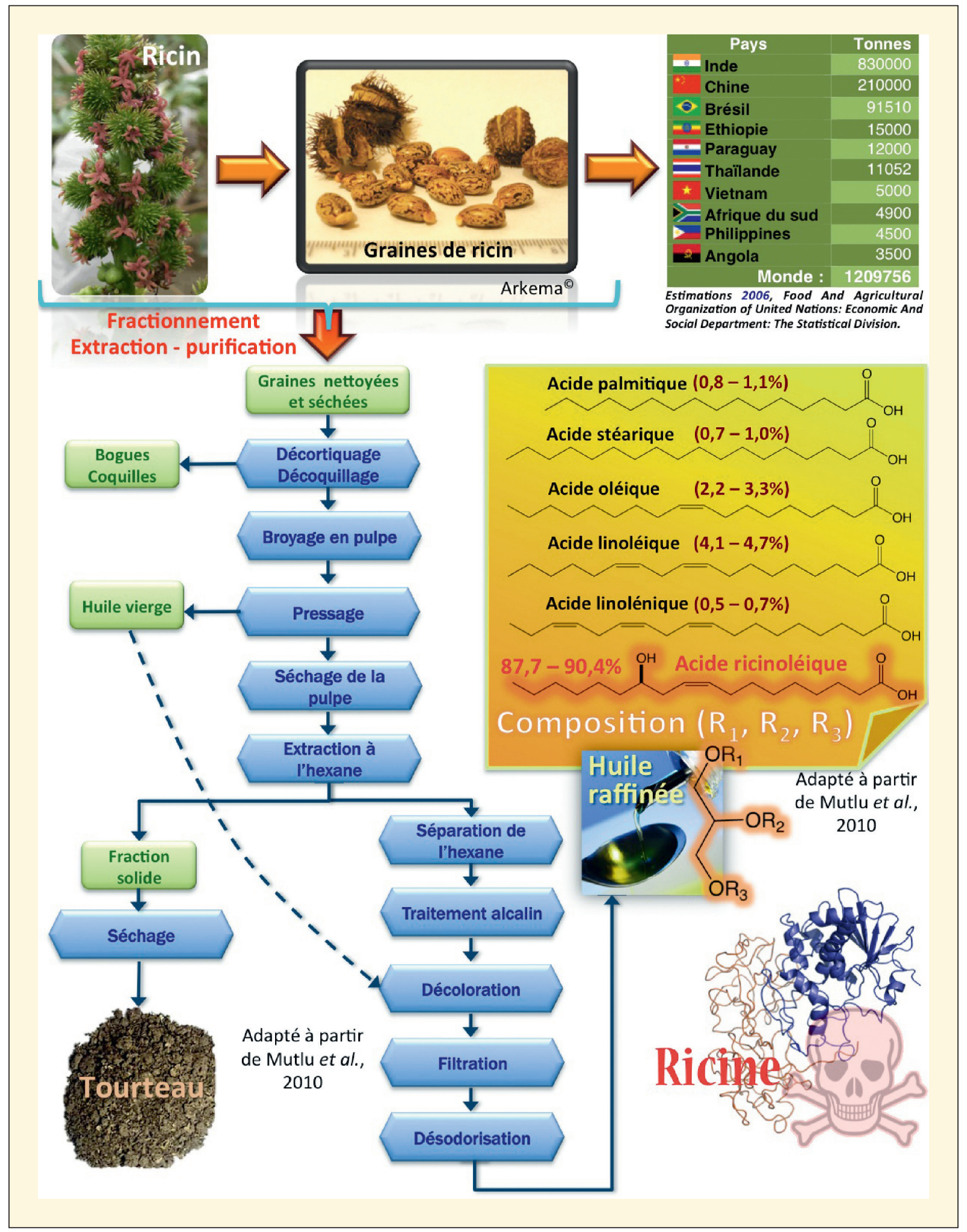

Figure 1. En haut à gauche: photo d'un plant de ricin, et de capsules \& graines de ricin; en haut à droite: statistiques de productions de graines de ricin dans le monde; en bas à gauche : exemple de procédé d'extraction de l'huile de ricin; au milieu à droite : composition de l'huile de ricin. 
figure 1 donne la production de graines de ricin telle que reportée pour l'année 2006. L'Inde y domine le marché mondial de manière écrasante, en fournissant près de $70 \%$ (830 kt/an) des graines disponibles dans le monde. Elle est suivie de la Chine qui, rappelons-le, a importé la culture du ricin depuis I'Inde il y a environ 1500 ans, avec $18 \%$ de la production mondiale $(210 \mathrm{kt} / \mathrm{an})$, soit près de quatre fois moins que I'Inde. Malgré ce deuxième rang, la production ne suffit pas à alimenter le marché interne et la Chine est un importateur net. Le troisième acteur significatif du secteur est le Brésil, avec environ $8 \%$ du marché mondial (91,5 kt/an), alors qu'il était à la première place il $y$ a une trentaine d'années. Les autres producteurs ne représentent chacun guère plus de $1 \%$ de la production mondiale. Notons que l'Égypte et l'Éthiopie, bien que berceaux historiques supposés du ricin, n'ont pas tiré partie par la suite de cette ressource potentielle de revenus et se cantonnent maintenant à de petits volumes, très largement dépassés par l'Inde et la Chine. Par ailleurs, notons que les États-Unis ont un temps été parmi les plus importants producteurs de ricin mais les circonstances économiques de 1972 les ont relégués au statut d'importateur et ils dépendent maintenant de l'étranger pour leur approvisionnement.

\section{Un marché volatile}

Le tableau 1 retrace l'évolution du prix de I'huile de ricin depuis 2002 jusque janvier 2010. L'extrême volatilité du marché y apparaît clairement, avec un prix très raisonnable en 2002 de 675 US $\$ / t$, lequel augmentera progressivement jusqu'en milieu d'année 2008 pour dépasser les 1300 US\$/t, avec un point culminant à 1500 US\$/t en juillet-août 2008 (tableau 2) soit largement le double du prix moyen observé en 2002. Ces fluctuations très importantes du coût de la matière première constituent un paramètre très important qui peut gréver de manière imprévisible l'équilibre économique de procédés de transformation avals, notamment par voie chimique.
Tableau 2. Valeur des graines, de l'huile et du tourteau de ricin lors du record de prix de juillet/ août 2008 - US\$/t, FOB Inde. (Données extraites de Castor Oil Report, février 2011, castoroil.in)

\begin{tabular}{|ll|}
\hline Produit & Prix \\
\hline Graine & 700 \\
\hline Huile & 1500 \\
\hline Tourteau & 110 \\
\hline
\end{tabular}

En outre, le tableau 2, même s'il est basé sur le cas particulier du point culminant de juillet-août 2008, montre que le prix à la tonne de l'huile de ricin est environ le double de celui de la graine, tandis que le tourteau vaut environ sept fois moins que la graine.

\section{Extraction et purification de l'huile de ricin}

Le procédé d'extraction de l'huile de ricin, présenté sur un schéma de la figure 1 (inspiré de Mutlu et Meier, 2010), n'est pas fondamentalement différent des procédés d'extraction conventionnels des huiles, à ceci près que le caractère " non-alimentaire » du ricin impose des conditions d'hygiène et de sécurité particulières, à savoir une ligne de production dédiée, complètement découplée des lignes de production d'huiles alimentaires pour éviter toute contamination éventuelle de ces dernières. Dans la suite, sont brièvement décrites les étapes d'extraction et de purification de l'huile dans un procédé opératoire " standard " qui peut donner lieu à des variantes, suivant le but recherché.

Après nettoyage des coques suivi d'une opération de décorticage/décoquillage, les graines ainsi obtenues sont broyées en pulpe et pressées à l'aide d'une presse à vis sans fin, afin d'en extraire I'huile de première pression qui peut être optionnellement filtrée. La fraction d'huile encore présente dans le résidu solide du pressage (tourteau composé des pulpes) est ensuite extraite à l'aide d'un solvant, conventionnellement I'hexane. L'huile d'extraction est alors récupérée par distillation. Les traitements avals de I'huile de ricin obtenue comprennent des étapes successives de dégommage (élimination notamment des composés susceptibles de devenir insolubles comme les phospholipides issus de la gangue externe des oléosomes, les lipoprotéines..., par hydrolyse en milieu acide par exemple), de neutralisation des acides gras libres (par traitement alcalin par exemple), de désodorisation (par distillation sous vide partiel en présence de vapeur d'eau), de décoloration (à l'aide d'un adsorbant, par exemple terres décolorantes comme la terre de foulon, charbon actif. ..), de filtration (élimination de cires, de débris...) et éventuellement de séparation, si l'on veut récupérer des composés spécifiques.

Revenons ici sur la toxicité du ricin. L'acide ricinoléique en lui-même possède une action purgative assez dangereuse pour l'organisme, mais c'est la ricine, protéine déjà évoquée plus haut, qui est la plus dangereuse. C'est une toxine redoutable, présente dans toute la plante, qui permet au ricin d'être référencé dans le Guinness Book des Records comme la plante la plus vénéneuse du monde. Et la nocuité du ricin ne s'arrête pas là car la plante contient aussi un allergène. . .

Cette toxicité du ricin fait de ses produits dérivés (huile, tourteau. . .) des composés de choix réservé à la chimie, les dangers d'une utilisation pour des applications alimentaires étant bien réels en cas de mauvaise manipulation. Mentionnons cependant les efforts de recherche effectués pour créer des variétés dépourvues de ricine.

\section{Nature, composition et propriétés de I'huile de ricin; quelques mots sur les sous-produits du procédé d'extraction- purification (figure 1)}

Les graines de ricin contiennent environ 50 70\% d'une huile, un triglycéride dont les chaînes d'acide gras sont

Tableau 1. Évolution du prix de I'huile de ricin (prix moyen pour un produit de qualité commerciale) - US\$/t, FOB Bombay. (Données extraites de Castor Oil Report, février 2011, castoroil.in)

\begin{tabular}{|lccccccccccc|}
\hline Année & $\mathbf{2 0 0 2}$ & $\mathbf{2 0 0 3}$ & $\mathbf{2 0 0 4}$ & $\mathbf{2 0 0 5}$ & $\mathbf{2 0 0 6}$ & $\mathbf{2 0 0 7}$ & $\begin{array}{l}\mathbf{2 0 0 8} \\
\text { (Fév.) }\end{array}$ & $\begin{array}{l}\mathbf{2 0 0 8} \\
\text { (Juin) }\end{array}$ & $\begin{array}{l}\mathbf{2 0 0 9} \\
\text { (Janv.) }\end{array}$ & $\begin{array}{l}\mathbf{2 0 0 9} \\
\text { (Juin) }\end{array}$ & $\begin{array}{l}\mathbf{2 0 1 0} \\
\text { (Janv.) }\end{array}$ \\
\hline Prix & 675 & 925 & 850 & 925 & 775 & 1025 & 1160 & 1350 & 1050 & 1104 & 1330 \\
\hline
\end{tabular}


composées à près de $90 \%$ d'acide ricinoléique [acide $(9 Z, 12 R)$-12-hydroxyoctadéc-9-énoique], ce qui est remarquable d'uniformité. Les acides oléique et linoléique sont les deux autres composés significatifs, bien que présents en bien moindres quantités : ils représentent respectivement environ 4 et $3 \%$ des chaînes d'acides gras. Les autres composés, très minoritaires, sont les acides palmitique, stéarique et linoléique, qui représentent chacun moins de $1 \%$. Selon les données de G. R. O'shea Company Vertelius, on trouve aussi de l'acide éicosanoique et de l'acide dihydrostéarique en très faibles quantités (non représentés sur la figure 1).

Cette uniformité de composition (grande majorité d'acide ricinoléique) confère des qualités spécifiques à I'huile de ricin que les autres huiles végétales ne possèdent pas. En effet, l'acide ricinoléique possède une particularité unique, à savoir la présence d'un groupement hydroxyle sur la chaîne grasse qui le distingue des autres acides gras naturels qui, eux, en sont dépourvus. Au niveau physicochimique, cette caractéristique induit quelques propriétés intéressantes et uniques. Ainsi, I'huile de ricin possède une grande affinité pour les surfaces métalliques (qualités de mouillage). Son point éclair est de seulement $229^{\circ} \mathrm{C}$, alors qu'elle ne s'enflamme qu'à $449{ }^{\circ} \mathrm{C}$. Par ailleurs, sa viscosité très élevée en fait I'huile végétale la plus dense.

En plus de conférer des propriétés particulières à l'huile de ricin, la présence d'un groupement hydroxyle ouvre des perspectives supplémentaires de transformations chimiques comme nous le verrons plus loin.

Concernant les sous-produits de la filière, ils sont issus du procédé d'extraction de I'huile et comprennent des coques, le tourteau, ainsi que des acides gras libres, des gommes, etc. La présence de débouchés et le développement d'applications pour ces sous-produits permettent de proposer un schéma intégré et ainsi d'accroître la rentabilté économique de la chaîne globale de transformation.

\section{L'huile de ricin, un composé aux multiples applications directes}

L'huile de ricin est utilisée comme standard absolu pour les mesures de viscosité grâce à ses propriétés physi- ques uniformes et stables dans le temps. La présence de groupements hydroxyles polaires permet à l'huile de ricin d'être directement compatible avec une grande variété de résines naturelles et synthétiques, de cires, de polymères et d'élastomères pour lesquels elle peut même servir de plastifiant. L'huile de ricin possède aussi d'excellentes propriétés comme émollient et lubrifiant, ainsi que de remarquables propriétés de mouillage et de dispersion de colorants, pigments et fillers. Dans cette section, nous allons décrire les applications directes de I'huile de ricin, lesquelles peuvent être encore diversifiées, comme nous le verrons dans la section suivante, par la synthèse de produits chimiques dérivés aux propriétés spécifiquement ajustées.

Ainsi, en plus des applications historiques décrites plus haut (cosmétiques, combustible pour les lampes), la suite présente un panel sérié d'applications directes :

1) comme lubrifiant: de par ses propriétés spécifiques, l'huile de ricin possède un grand intérêt comme lubrifiant. Elle fut utilisée par le passé pour l'aviation, mais aussi dans les moteurs de véhicules personnels, et plus spécifiquement dans les moteurs de voitures et motos de course ainsi que dans les moteurs - plus particulièrement deux temps - de modèles réduits, secteur dans lequel elle alimente encore les débats. Dans cette application, malgré une excellente tenue à haute température, elle possède cependant l'inconvénient d'une oxydation relativement rapide qui donne lieu à la production de gommes (d'où une utilisation plutôt dans les moteurs de véhicules de compétition ou de modèles réduits pour lesquels le démontage et le remontage du moteur sont fréquents). Ces gommes sont réputées néfastes par leur action d'encrassement des moteurs, même si certains soulignent leurs propriétés d'étanchéisation par dépôt sur la surface interne des pistons. Notons que cette propriété pourrait s'avérer très intéressante dans le cas de moteurs rotatifs (en général à cycle de Robert et Wankel), dont I'un des principaux soucis est justement l'étanchéité des segments des chambres trochoïdes. Ainsi, I'huile de ricin a progressivement cédé sa place à I'utilisation d'huiles minérales et synthétiques, mais on la retrouve parfois en mélange avec ces dernières (surtout dans les modèles réduits) ou sous une forme modifiée chimiquement pour pallier ses inconvénients tout en continuant de bénéficier de ses propriétés remarquables;

2) en pharmacie : comme indiqué plus haut, les vertus laxatives de l'huile de ricin sont connues depuis très longtemps. Cependant, son utilisation pour cette application s'avérant dangereuse en raison d'effets violents, son usage à effet laxatif est maintenant formellement proscrit. En pharmacie, ce sont maintenant plutôt ses propriétés en tant qu'excipient qui sont utilisées, même si I'huile de ricin est officiellement classifiée dans les " excipients à effet notoire » et ainsi déconseillée, par exemple, aux enfants de moins de trois ans, en raison de possibilités de troubles digestifs, d'eczéma en application locale, de bouffées de chaleur, de difficultés à respirer, et de chute de tension dans le cas d'administration par injection ;

3) dans I'alimentation : I'huile de ricin est référencée comme additif alimentaire dans le Codex Alimentarius sous le numéro 1503, avec les catégories fonctionnelles suivantes : antiagglomérant, substance inerte, émulsifiant et agent de glaçage. Par ailleurs, la NGAA (Norme générale codex pour les additifs alimentaires) autorise des concentrations maximales de $2100 \mathrm{mg} / \mathrm{kg}$ dans les chewing-gums, $350 \mathrm{mg} / \mathrm{kg}$ dans le cacao et les produits chocolatés, $500 \mathrm{mg} / \mathrm{kg}$ dans les pâtisseries et les bonbons, et $1000 \mathrm{mg} / \mathrm{kg}$ dans les compléments alimentaires. II semblerait par ailleurs qu'en Inde il soit courant de mélanger jusqu'à $3 \%$ d'huile de ricin avec les huiles végétales comestibles.

Enfin, mentionnons que le tourteau de ricin est employé en horticulture. En effet, outre ses qualités nutritives (engrais azoté à minéralisation lente, riche en matière organique et en minéraux), il possède des propriétés nématicides et insecticides, en plus de son pouvoir répulsif contre les rongeurs. Il est en revanche à utiliser avec prudence car il peut s'avérer un poison mortel pour les animaux domestiques qui viendraient à l'ingérer.

\section{Valorisation chimique du ricin et des principaux coproduits de la filière}

Les trois fonctionnalités présentes dans I'huile de ricin (groupement hydroxyle, double liaison et fonction ester) auto- 


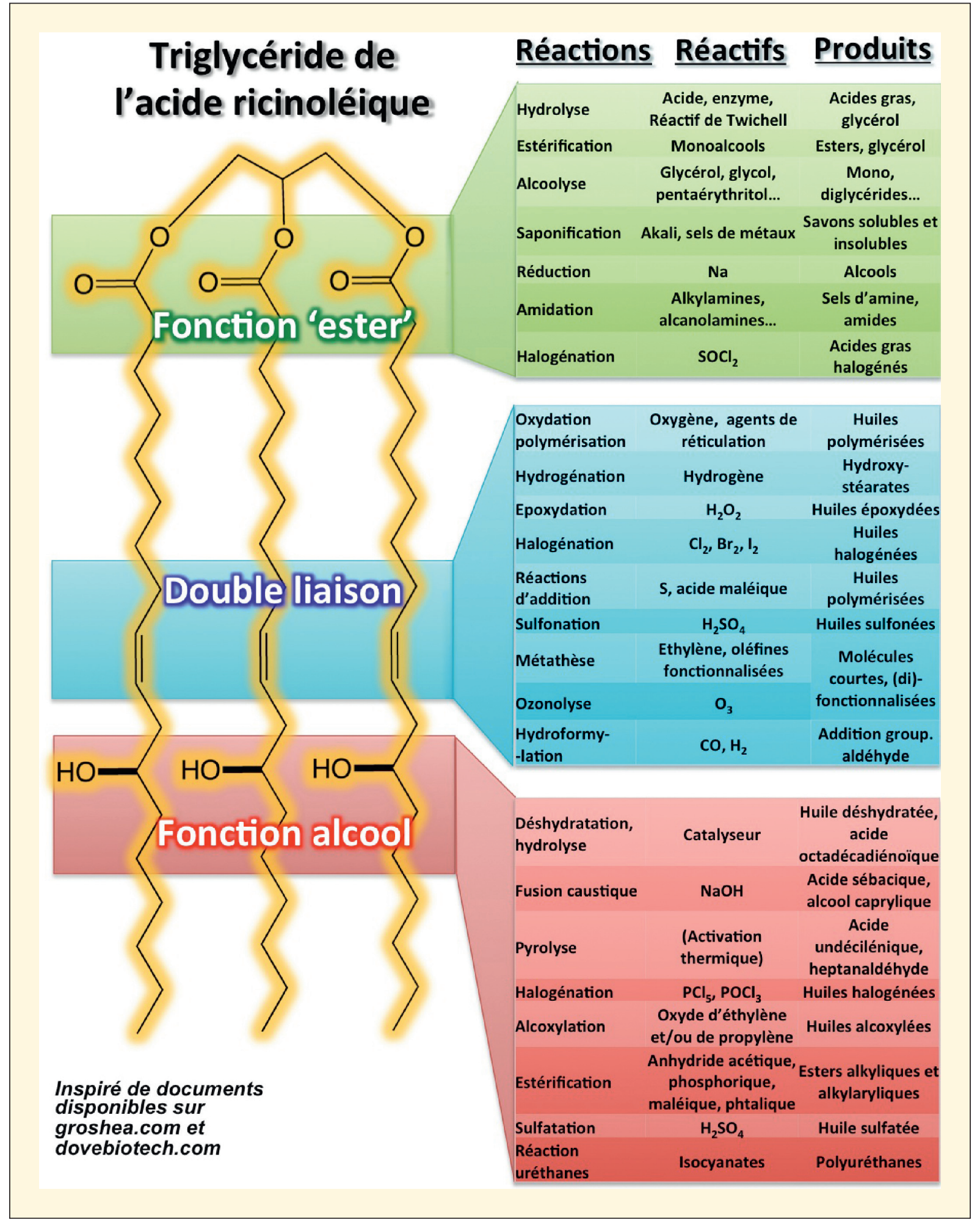

Figure 2. Exemples de transformations chimiques possibles à partir des trois fonctionnalités présentes dans l'huile de ricin (triglycéride de l'acide ricinoléique). 
risent de nombreuses variations par transformations chimiques. La figure 2 détaille un grand nombre de possibilités de réactions qui font, pour la majorité, appel à des procédés basés sur la catalyse. Bien entendu, rien n'empêche de modifier séquentiellement de manière sélective la nature chimique des différentes fonctions, ce qui ouvre d'autant plus de perspectives vers tout un éventail de composés chimiques plurifonctionnalisés par rapport à la molécule de départ.

Ces transformations peuvent être effectuées aussi, par exemple, à partir de I'ester méthylique obtenu par transestérification préalable à l'aide de méthanol, qui sert à cliver la chaîne grasse du squelette de glycérol (réaction d'obtention du biodiesel).

Concernant le principal sous-produit de la filière, le tourteau, il peut aussi subir diverses transformations en vue de sa valorisation. II peut être détoxifié par autoclavage en vue d'applications alimentaires pour les animaux, par exemple. On peut aussi envisager sa gazéification en syngas pour ensuite éventuellement effectuer une réaction Fischer-Tropch pour donner des alcanes ou une conversion syngas pour donner des alcools. La conversion en biogaz pour obtenir du $\mathrm{CH} 4$ est aussi une option qui permet de valoriser le tourteau.

Malgré un nombre conséquent d'applications possibles (voir les nombreux exemples sur la figure 2), il ne faut pas oublier que l'aura " verte " d'un produit chimique est loin d'en assurer à elle seule le succès, et n'a de répercussion que si elle est assortie d'un intérêt économique avéré et d'une analyse de cycle de vie attestant cette vertu, et que ce produit apporte un plus technique qui lui donne un avantage sur ses concurrents pétro-sourcés. C'est par exemple le cas de deux composés commercialisés respectivement par Arkema et Rhodia :
1) Rilsan ${ }^{\circledR}$. Arkema communique sur un polymère issu du végétal, le polyamide 11 ("PA11 ") dont ils maîtrisent la synthèse depuis le ricin à l'aide d'une technologie développée en 1942, et qu'ils ont commencé à commercialiser un peu plus tard à partir de 1947. Notons qu'Arkema possède un portefeuille de matériaux issus partiellement de I'huile de ricin, comme le Pebax ${ }^{\circledR}$ Rnew ou le Platamid ${ }^{\mathbb{R}}$ Rnew. Arkema déclare par ailleurs utiliser les sousproduits du procédé de production du Rilsan ${ }^{\mathbb{R}}$, par exemple dans l'industrie du parfum ou des cosmétiques, l'industrie alimentaire, pharmaceutique ou du cuir, dans les lubrifiants, le bâtiment, cette intégration devant équilibrer l'intérêt économique de cette filière. Selon Arkema, les avantages du Rilsan résident non pas tant dans une propriété donnée où il excellerait, mais dans un compromis remarquable entre ses propriétés thermiques, chimiques et mécaniques. Ses qualités techniques et sa résistance en font un composé d'intérêt dans des applications à forte valeur ajoutée, telle que les lignes essence dans les voitures ou les pipes offshore.

2) PA6.10. C'est un autre polyamide, basé celui-ci en partie sur l'huile de ricin comme matière première (utilisation de I'acide sébacique dérivé), le PA6.10, que Rhodia commercialise. Rhodia communique sur l'empreinte environnementale réduite de son PA6.10 qui permettrait, à performances équivalentes, de réduire la consommation de matières premières fossiles de $20 \%$ par rapport à un polyamide entièrement pétrosourcé. Ici aussi, l'argument mis en avant par Rhodia, en plus de l'avantage écologique, est la haute performance des plastiques dérivés qui élargissent le champ des débouchés du polyamide à des applications de haute technicité. En effet, le PA6.10 possède de bonnes performances mécaniques et thermiques (point de fusion de $215^{\circ} \mathrm{C}$ ), une résistance chimique comparable à celle du PA11 et du PA12, un très haut niveau de propriétés barrières aux essences, et une faible reprise à l'humidité, cette dernière étant intermédiaire entre celle du PA6 et celle du PA12. Ainsi, les applications citées en exemple par Rhodia sont la fabrication de tubes souples pour le marché des systèmes de contrôle et d'assistance pneumatique et pour la fabrication de conduits et de raccords pour le marché des systèmes d'alimentation d'essence des moteurs.

\section{Conclusion}

Bonne qualité et grande homogénéité de composition (90\% de chaînes d'acide ricinoléique) font de l'huile de ricin un composé de choix pour l'industrie chimique. Nous avons vu que la versatilité du triglycéride de l'acide ricinoléique autorise de nombreuses variations par transformation chimique, ce qui ouvre de nombreuses perspectives d'applications. L'huile de ricin possède ainsi un potentiel de développement conséquent dans le secteur de la chimie. Ce potentiel peut être cependant limité par une certaine incertitude, laquelle réside dans la capacité d'approvisionnement du marché et dans les fluctuations importantes des cours. La diversification des sources de production et l'augmentation des surfaces cultivées semblent être une clef importante pour libérer tout le potentiel économique de ce composé et ainsi en déverrouiller complètement les perspectives d'utilisation comme matière première renouvelable pour l'industrie chimique.

\section{REFERENCE}

Mutlu H, Meier MAR. Castor oil as a renewable resource for the chemical industry. Eur J Lipid Sci Technol 2010 ; 112 : 10-30. 